\title{
First report of turnip mosaic virus infecting Perilla frutescens in Korea
}

\author{
Suk-Hyun Park ${ }^{1}$ Ji-Soo Park ${ }^{1}$ - Tae-Seon Park ${ }^{1}$ Jin-Sung Hong ${ }^{1}$ \\ Received: 27 August 2019 / Accepted: 28 February 2020 / Published online: 16 March 2020 \\ (C) Società Italiana di Patologia Vegetale (S.I.Pa.V.) 2020
}

Keywords Turnip mosaic virus $\cdot$ Perilla frutescens $\cdot$ Sesame

Perilla frutescens (wild sesame) is a member of the family Lamiaceae. It is used as an edible oilseed crop in Korea. Although $P$. frutescens is cultivated commonly in Korea, little is known about the viruses that infect this species (Cho et al. 2005). During a survey in May 2016, virus-like symptoms were observed on $70-80 \%$ of $P$. frutescens plants on a farm located in Chuncheon, Korea. Symptoms included mild mosaic and yellowing symptoms. To identify the causative virus, total RNA was extracted from the leaves of affected $P$. frutescens and seven genus-specific primer sets for Ilarvirus, Potyvirus, Fabavirus, Tospovirus, Potexvirus, Tobamovirus and Cucumovirus detection were used for RT-PCR. A positive result was only obtained with the Potyvirus specific primers (Zheng et al. 2008). Nicotiana benthamiana and N. tabacum cv. Xanthi nc were mechanically inoculated from affected P. frutescens and observed for symptoms. Necrotic local lesions were induced on the inoculated leaves of $N$. tabacum $\mathrm{cv}$. Xanthi nc, suggesting turnip mosaic virus (TuMV) infection. To confirm the presence of TuMV and determine the full-length sequence of this isolate, we designed six pairs of primers targeting conserved regions of the TuMV genome. Using these primers, we determined the complete sequence of an isolate of TuMV named TuMV-KPF (GenBank accession No. LC413507). Four indicator plant species (five plants each) were mechanically inoculated with TuMV-KPF to examine

Electronic supplementary material The online version of this article (https://doi.org/10.1007/s42161-020-00536-z) contains supplementary material, which is available to authorized users.

Jin-Sung Hong

jinsunghong@kangwon.ac.kr

1 Department of Bio-resources Sciences, College of Agriculture and Life Sciences, Kangwon National University, Chuncheon 24341, South Korea the biological properties: systemic mosaic symptoms were induced on N. benthamiana and Raphanus sativus; $N$. tabacum cv. Xanthi nc showed necrotic spots on inoculated leaves while four plants of Brassica rapa subsp. pekinensis did not show symptoms, but one developed necrosis. BLASTn analysis indicated that TuMV-KPF had $99.31 \%$ and $99.25 \%$ nucleotide identity with radish isolates TuMV-CCLB (KR153038) and TuMV-TANX2 (EU734433), respectively. Furthermore, phylogenetic analysis revealed that TuMV-KPF belongs to the basal-BR group of strains that comprise of brassica and nonbrassica infecting isolates from Eurasia (Ohshima et al. 2002). To our knowledge, this is the first report of TuMV infection in P. frutescens in Korea or elsewhere.

Funding This research was financially supported by Cooperative Research Program for Agricultural Science \& Technology Development (Project No. PJ014507032019).

\section{References}

Cho J-D, Kim J-S, Kim J-Y, Kim J-H, Lee S-H, Choi G-S, Kim H-R, Chung B-N (2005) Occurrence and symptoms of tomato spotted wilt virus on vegetables in Korea (I). Res Plant Dis 11(2):213-216

Ohshima K, Yamaguchi Y, Hirota R, Hamamoto T, Tomimura K, Tan Z, Sano T, Azuhata F, Walsh JA, Fletcher J, Chen J, Gera A, Gibbs A (2002) Molecular evolution of Turnip mosaic virus: evidence of host adaptation, genetic recombination and geographical spread. J GenVirol 83:1511-1521

Zheng L, Gibbs MJ, Rodoni BC (2008) Quantitative PCR measurements of the effects of introducing inosines into primers provides guidelines for improved degenerate primer design. J Virol Methods 153:97-103

Publisher's note Springer Nature remains neutral with regard to jurisdictional claims in published maps and institutional affiliations. 